\title{
Relaxation and Coarsening Dynamics in Superconducting Arrays
}

\author{
Gun Sang Jeon, ${ }^{1,2}$ Sung Jong Lee, ${ }^{3}$ and M.Y. Choi ${ }^{4}$, \\ ${ }^{1}$ Center for Strongly Correlated Materials Research, \\ Seoul National University, Seoul 151-747, Korea \\ ${ }^{2}$ Department of Physics and Institute for Basic Science Research, \\ Sung Kyun Kwan University, Suwon 440-746, Korea \\ ${ }^{3}$ Department of Physics and Center for Smart Bio-Materials, \\ The University of Suwon, Kyunggi-do 445-743, Korea \\ ${ }^{4}$ Department of Physics, Seoul National University, Seoul 151-747, Korea
}

\begin{abstract}
We investigate the nonequilibrium coarsening dynamics in two-dimensional overdamped superconducting arrays under zero external current, where ohmic dissipation occurs on junctions between superconducting islands through uniform resistance. The nonequilibrium relaxation of the unfrustrated array and also of the fully frustrated array, quenched to low temperature ordered states or quasi-ordered ones, is dominated by characteristic features of coarsening processes via decay of point and line defects, respectively. In the case of unfrustrated arrays, it is argued that due to finiteness of the friction constant for a vortex (in the limit of large spatial extent of the vortex), the typical length scale grows as $\ell_{s} \sim t^{1 / 2}$ accompanied by the number of point vortices decaying as $N_{v} \sim 1 / t$. This is in contrast with the case that dominant dissipation occurs between each island and the substrate, where the friction constant diverges logarithmically and the length scale exhibits diffusive growth with a logarithmic correction term. We perform extensive numerical simulations, to obtain results in reasonable agreement. In the case of fully frustrated arrays, the domain growth of Ising-like chiral order exhibits the low-temperature behavior $\ell_{q} \sim t^{1 / z_{q}}$, with the growth exponent $1 / z_{q}$ apparently showing a strong temperature dependence in the low-temperature limit.
\end{abstract}

PACS numbers: 74.50+r, 67.40.Fd

\section{INTRODUCTION}

In a statistical system quenched from the random disordered state to a low-temperature state below the transition temperature, the average length scale $\ell$ of ordered domains typically grows in time as a power law $\ell \sim t^{1 / z}$, where the growth exponent $1 / z$ depends on the dimension of the space and of the relevant order parameter, in addition to the conserved or nonconserved nature of the latter in the relaxation dynamics. Depending crucially on the dimension of the order parameter, characteristic topological defects such as point vortices or domain walls are generated in the initial disordered state and the annihilation of these defects gives the main mechanism of coarsening and phase ordering in the system. The observed self-similarity of these coarsening systems at different time instants, is embodied in the so-called dynamic scaling hypothesis of the equal-time spatial correlation function of the order parameter.

One of the extensively studied cases is the system with the order parameter dimension $n=2$ in two spatial dimensions $d=2$, i.e., the two-dimensional (2D) $X Y$ model, , the relevant topological defect is the point vortex 2 3. A 4 A convenient physical realization of the $2 \mathrm{D}$ $X Y$ model can be found in the $2 \mathrm{D}$ regular arrays of superconducting Josephson junctions, where the phases of the superconducting order parameters correspond to the $X Y$ spins. In such a superconducting array, frustration may be induced simply by applying an external magnetic field.6 It affects thermodynamic properties in a crucial way, leading to a variety of phase transitions.
In view of the remarkable diversity in the equilibrium properties, also expected for the system are a variety of interesting dynamic behaviors, particularly in relaxation toward equilibrium. The quasi-long-range order present in the 2D pure $X Y$ model 1 describing the array without frustration (i.e., in the absence of a magnetic field), is expected to exhibit coarsening through annihilation of vortex-antivortex pairs, giving rise to characteristic algebraic relaxation. The vortex dynamics and the resulting scaling behavior in the 2D $X Y$ model has been an issue for some time, concerning late-time scaling and true nature of the asymptotic growth law. It is also of interest to examine the effects of the additional long-range order on the relaxation in the fully frustrated array (with half the flux quantum per plaquette) 9 . where the relaxation dynamics is expected to be dominated by coarsening processes accompanying decay of domain walls and also point defects; the latter corresponds to the corners on the domain walls.

The main purpose of this paper is to probe the properties of the nonequilibrium relaxation dynamics associated with coarsening processes in the unfrustrated and fully frustrated superconducting arrays under rapid quenching from the high-temperature disordered state to the low-temperature ordered or quasi-ordered states. We would like to understand what kind of specific features emerge in the relaxation dynamics of these systems governed by the resistively-shunted-junction (RSJ) dynamic equations, especially in connection with the coarsening processes. The time-dependent behaviors of various onetime and two-time correlations involving energy and or- 
der parameters such as vortex density and chirality are investigated numerically.

In the case of an unfrustrated array, we argue that the friction constant of a slow moving vortex remains finite in the limit of large extent of the vortex:10 this leads (via a simple force-velocity relation) to a power-law growth of length scales with growth exponent $1 / 2$, together with the excess number of vortices decaying as $\tilde{N}_{v} \sim t^{-\alpha_{v}}$ with $\alpha_{v}=1$. Extensive simulations indeed show that $\alpha_{v}$ takes a value that is very close to unity in a wide range of temperatures. This is in contrast to the simulation results of the ordinary phase ordering process based on either Monte Carlo algorithms or simple Langevin dynamics of the $X Y$ model (see section III for details), where a logarithmic correction factor is observed in the time dependence of the growing length scale. The latter case corresponds to the situation in Josephson-junction arrays where dissipation occurs between each island and the substrate.

In relation to the coarsening dynamics of fully frustrated arrays, Lee, et al 11 performed dynamic simulations on the fully frustrated $X Y$ models, based on the simple Langevin equations for $X Y$ phase angles. It was shown that the domain growth exponents are temperature-dependent and that there exist two regimes of domain-growth morphology. Later, coarsening was also studied in the dual representation, i.e., the lattice Coulomb gas of fractional vortices, through the use of Monte Carlo methods on the vortex degrees of freedom.12 The latter work, even though based on different dynamics, confirmed most features of the previous results and gave some numerical evidence for unbinding of the socalled single-step kinks at a finite temperature. Those rich features of coarsening in the fully frustrated $X Y$ model can be partly attributed to the existence of the Ising-like chiral degrees of freedom in addition to the underlying phase degrees of freedom. Here, in this work, we perform dynamic simulations on the coarsening of the fully frustrated Josephson-junction arrays, governed by the RSJ equations, and examine whether temperaturedependent coarsening also emerges. It is found that, similarly to the previous works, the domain growth exponents are strongly temperature-dependent: The exponents are proportional to the temperature in the low-temperature limit and increase monotonically with the temperature.

\section{EQUATIONS OF MOTION}

We begin with the set of equations of motion for the phases $\left\{\phi_{i}\right\}$ of the superconducting order parameters in an $L \times L$ square array. In the RS.Jmodel with the fluctuating twist boundary conditions, 13 they read:

$$
\sum_{j}^{\prime}\left[\frac{d \widetilde{\phi}_{i j}}{d t}+\sin \left(\widetilde{\phi}_{i j}-\mathbf{r}_{i j} \cdot \boldsymbol{\Delta}\right)+\zeta_{i j}\right]=0
$$

where we have employed the abbreviations $\widetilde{\phi}_{i j} \equiv$ $\phi_{i}-\phi_{j}-A_{i j}$ and $\mathbf{r}_{i j} \equiv \mathbf{r}_{i}-\mathbf{r}_{j}$, and the primed summation runs over the nearest neighbors of grain $i$. The position of grain $i$ is represented by $\mathbf{r}_{i}=\left(x_{i}, y_{i}\right)$ with the lattice constant set equal to unity while the gauge field $A_{i j}$ is given by the line integral of the vector potential $\mathbf{A}$ :

$$
A_{i j} \equiv \frac{2 \pi}{\Phi_{0}} \int_{\mathbf{r}_{i}}^{\mathbf{r}_{j}} \mathbf{A} \cdot d \mathbf{l}
$$

with the flux quantum $\Phi_{0} \equiv h c / 2 e$. The frustration parameter $f$, which measures the number of flux quanta per plaquette, is given by the directional sum of the gauge field $A_{i j}$ around a plaquette:

$$
f \equiv \frac{1}{2 \pi} \sum_{P} A_{i j}
$$

In Eq. (11) the energy and the time have been expressed in units of $\hbar I_{c} / 2 e$ and $\hbar / 2 e R I_{c}$, respectively, with singlejunction critical current $I_{c}$ and shunt resistance $R$. The thermal noise current $\zeta_{i j}$ is assumed to be white, satisfying

$$
\left\langle\zeta_{i j}(t+\tau) \zeta_{k l}(t)\right\rangle=2 k_{B} T \delta(\tau)\left(\delta_{i k} \delta_{j l}-\delta_{i l} \delta_{j k}\right)
$$

at temperature $T$. Henceforth we set the Boltzmann constant $k_{B} \equiv 1$. The dynamics of the twist variables $\boldsymbol{\Delta} \equiv\left(\Delta_{x}, \Delta_{y}\right)$ is governed by

$$
\frac{d \Delta_{a}}{d t}=\frac{1}{L^{2}} \sum_{\langle i j\rangle_{a}} \sin \left(\widetilde{\phi}_{i j}-\Delta_{a}\right)+\zeta_{a},
$$

where $\sum_{\langle i j\rangle_{a}}$ denotes the summation over all nearestneighboring pairs in the $a$-direction $(a=x, y)$ and $\zeta_{a}$ satisfies

$$
\left\langle\zeta_{a}(t+\tau) \zeta_{b}(t)\right\rangle=\frac{2 T}{L^{2}} \delta_{a b} \delta(\tau) .
$$

Note that in equilibrium the above set of equations of motion leads to a Gibbs distribution with the Hamiltonian for the frustrated $X Y$ model

$$
H=-\sum_{\langle i, j\rangle} \cos \left(\tilde{\phi}_{i j}-\mathbf{r}_{i j} \cdot \Delta\right),
$$

where the fluctuating twist boundary conditions have been incorporated. In numerical simulations, we have integrated directly the equations of motions in Eqs. (11) and (5) via the modified Euler method with time steps $\Delta t=0.05$. Simulations have been performed on square arrays with linear size $L=400$ (unfrustrated arrays) and $L=128$ (fully frustrated arrays).

To study the relaxation and coarsening in the superconducting arrays, we let the systems evolve from random initial configurations at given temperatures and measure the following quantities: 
1. The excess amount of topological defects such as vortices and domain walls

$$
\tilde{N}(t) \equiv N(t)-N(\infty),
$$

where $N(t)$ is the total amount (number or length) at time $t$ and $N(\infty)$ refers to the amount in equilibrium. [In the case of vortices $N$ denotes the sum of the total number of vortices (plus charges) and that of antivortices (minus charges).] It is understood that the appropriate ensemble average over random initial configurations is to be taken.

2. Excess energy relaxation defined to be

$$
\tilde{E}(t) \equiv E(t)-E(\infty)
$$

where the energy (per site) $E(t)$ at time $t$ is given by

$E(t) \equiv-\frac{1}{L^{2}} \sum_{\langle i, j\rangle}\left\langle\cos \left[\widetilde{\phi}_{i j}(t)-\mathbf{r}_{i j} \cdot \boldsymbol{\Delta}(t)\right]\right\rangle$

with the summation over all nearest-neighboring pairs and $\langle\cdots\rangle$ denoting the ensemble average over random initial configurations.

3. The equal-time spatial correlation function

$$
C(r, t)=\left\langle O^{*}(\mathbf{r}, t) O(0, t)\right\rangle
$$

of the order parameter $O(\mathbf{r}, t) \equiv e^{i \phi(\mathbf{r}, t)}$.

According to the dynamic scaling hypothesis, the correlation function assumes the 2D scaling form

$$
C(r, t)=r^{-\eta} g(r / \ell)
$$

with the appropriate scaling function $g(x)$, where $\ell=\ell(t)$ is the typical length scale of ordered domains (at time $t$ ). While the exponent $\eta$ vanishes $(\eta=0)$ in the usual noncritical quenching to long-range ordered states, it takes nonzero values in the case of quenching to critical states, depending on the temperature $T$, i.e., $\eta=\eta(T)$.

\section{COARSENING AND VORTEX DYNAMICS IN UNFRUSTRATED ARRAYS}

It is well known that the unfrustrated system $\left(A_{i j}=\right.$ 0 ), described by the $X Y$ model in equilibrium, is critical below the Berezinskii-Kosterlitz-Thouless transition temperature $T_{c}(\approx 0.89) .14$ Previous works on relaxation of the $X Y$ model dealt mostly with the time-dependent Ginzburg-Landau equations (either the soft-spin version or the hard-spin version). The hard-spin version, where the magnitude of each spin is fixed, is described by the simple Langevin equations for phases:

$$
\frac{d \phi_{i}}{d t}=-\frac{\delta H}{\delta \phi_{i}}+\zeta_{i}
$$

where $H=-\sum_{\langle i, j\rangle} \cos \left(\phi_{i}-\phi_{j}\right)$ is the Hamiltonian and the noise $\zeta_{i}$ satisfies $\left\langle\zeta_{i}(t+\tau) \zeta_{j}(t)\right\rangle=2 T \delta_{i j} \delta(\tau)$. Experimentally, this simple Langevin dynamics corresponds to the situation where dissipation through the resistance between superconducting islands and the substrate dominates over those through the junction resistance.

In this model with no frustration, it was argued that the friction constant $\gamma$ of a slow moving vortex depends logarithmically on the size (spatial extent) $r$ of the vortex, i.e., $\gamma \sim \ln \left(r / r_{0}\right)$, where the cutoff length scale $r_{0}$ may be taken as the size of the vortex core. 15 Combining this result with the coulomb force law of $F(r) \sim-r^{-1}$, we get the relation $\dot{r} \ln \left(r / r_{0}\right) \sim-r^{-1}$, which leads to the conclusion that the time dependence of the vortex number exhibits logarithmic correction to the power law: $N_{v} \sim t^{-1} \ln t$. The typical length scale, e.g., the serpa ration between vortices, is then expected to follow 0 . 15 $\ell_{s} \sim(t / \ln t)^{1 / 2}$. Numerical confirmation of this prediction has turned out rather tricky and subtle. 16

Here, we apply a similar argument to the coupled RSJ dynamics of the superconducting arrays. Interestingly, the mobility of a vortex whose motion is governed by the RSJ dynamics is found not to vanish in the largeseparation limit $(r \rightarrow \infty)$ but to remain finite. This can be easily seen from the following argument based on the equations of motion in Eq. (1) for the phase variables. Equation (11) can be rewritten in the following form 17

$$
\sum_{j} M_{i j} \frac{d \phi_{j}}{d t}=-\frac{\delta H}{\delta \phi_{i}}-\sum_{j}^{\prime} \zeta_{i j}
$$

where $-M_{i j}$ is the discrete Laplacian matrix and the Hamiltonian $H$ is given by Eq. (7). In order to obtain the mobility of an isolated vortex, we compute the energy dissipation for an isolated vortex moving with a constant velocity $v$ along, say, the $x$ direction. The corresponding phase configuration takes the form $\phi_{v}(x-v t, y)$, where $\phi_{v}(x, y)$ represents the phase at site $i \equiv(x, y)$ in the presence of an isolated (static) vortex. At low temperatures, where the noise term may be neglected, we use Eq. (14) and write the energy dissipation in the form 15

$$
\frac{d E}{d t}=\sum_{i} \frac{\delta H}{\delta \phi_{i}} \frac{d \phi_{i}}{d t}=-\sum_{i j} M_{i j} \frac{d \phi_{j}}{d t} \frac{d \phi_{i}}{d t} .
$$

Inserting the vortex solution $\phi_{v}(x-v t, y)$ in the above equation and working in the continuum notation, we get the following expression for the energy dissipation

$$
\frac{d E}{d t}=-\gamma_{v} v^{2}
$$

where the friction constant (or the inverse of the mobility) $\gamma_{v}$ is given by

$$
\gamma_{v} \approx-\int d x d y \partial_{x} \phi_{v}(x-v t, y) \nabla^{2} \partial_{x} \phi_{v}(x-v t, y) .
$$

In the derivation of Eq. (17) from Eq. (15), it has been noted that in the continuum limit, the discrete Laplacian 


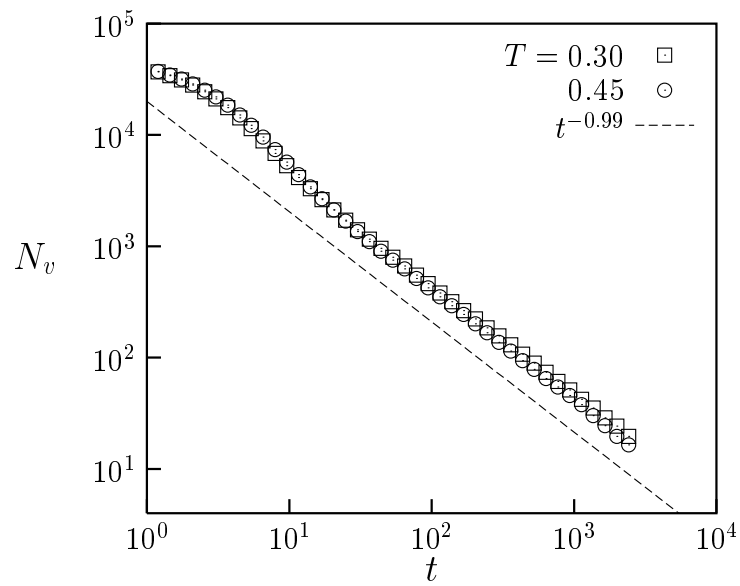

(a)

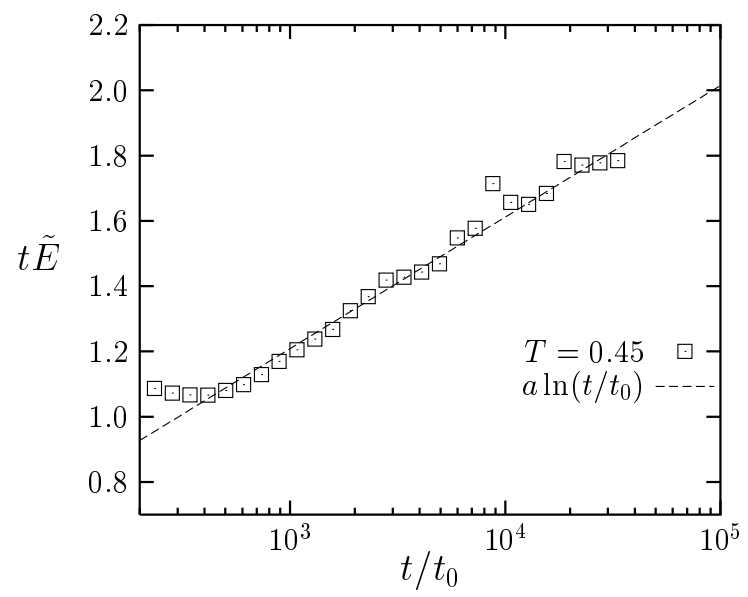

(b)

FIG. 1: Relaxation of (a) the vortex number $N_{v}$ at temperature $T=0.30$ and 0.45 and of (b) the excess energy $\tilde{E}$ at $T=0.45$ in the unfrustrated array of size $L=400$. In (a) the power-law behavior $t^{-0.99}$ is also plotted whereas (b) displays the best fit to the form $\tilde{E}=a t^{-1} \ln \left(t / t_{0}\right)$ with $a=0.175$ and $t_{0}=0.06$.

matrix $-M_{i j}$ turns into $\nabla^{2}$ and the time derivative becomes velocity multiplied by the spatial derivative with respect to $x$ for the ansatz vortex configuration. In the limit of zero vortex velocity $(v \rightarrow 0)$, one can easily see from dimensional argument that the mobility becomes finite for a vortex of an infinite extent. Namely, the above integral for $\gamma_{v}$ is convergent in the large-size limit, owing to the presence of the additional Laplacian derivative term $\nabla^{2}$. Since we have $\partial_{x} \phi_{v}(x, y) \sim r^{-1}$ for a single isolated vortex, the integrand in the above equation goes as $\sim r^{-4}$, which is infrared convergent in two dimensions.

Since the friction constant converges quickly to a finite value $\gamma_{0}$ as the extent of the vortex is increased, the equation of motion resulting from the force balance reads

$$
\gamma_{0} \dot{r} \sim-r^{-1}
$$

from which one can estimate the annihilation time for a vortex-antivortex pair of size $\xi$ to be $t \sim\left(\xi / \xi_{0}\right)^{2}$, with the cutoff length scale $\xi_{0}$ corresponding to the size of the vortex core. This can be interpreted as the growth law for the typical (average) separation between vortices as $\xi \sim t^{1 / 2}$, which in turn leads to the vortex number $N_{v} \sim \xi^{-2} \sim t^{-1}$. Figure 11(a) shows the relaxation of the number of vortices at temperatures $T=0.3$ and 0.45 . Observed in the late-time regime are good fits with $N_{v} \sim$ $t^{-0.99}$ and $t^{-0.98}$ at $T=0.45$ and at $T=0.3$, respectively, in excellent agreement with the expected $t^{-1}$ behavior.

This result may be used to obtain the energy relaxation behavior: On the basis of the dynamic scaling hypothesis and the Porod law, 1 which refers to the power-law singularity in the short (scaled) distance limit of the equal-time spatial correlation function due to the presence of topological defects, it is known that the excess energy for the $O(n)$ model with $n=2$ scales ast

$$
\tilde{E} \sim \ell^{-2} \ln \left(\frac{\ell}{r_{0}}\right)
$$

where the typical length scale may be taken to be separation between vortices $(\ell \approx \xi)$ and the cutoff to be the vortex core size $\left(r_{0} \approx \xi_{0}\right)$ in our system. Noting the simulation result $\xi \sim t^{1 / 2}$ presented above, we get $\tilde{E} \sim t^{-1} \ln \left(t / t_{0}\right)$ with $t_{0}$ being approximately the initial microscopic time scale taken by a vortex to move a single lattice spacing. The excess energy versus time measured at $T=0.45$ is shown in Fig. 11(b) and indeed fitted quite well to the expected form $\tilde{E} \approx a t^{-1} \ln \left(t / t_{0}\right)$ with $a=0.17$ and $t_{0}=0.06$. We have also tried powerlaw fitting of the form $\tilde{E} \sim t^{-\alpha}$, to obtain the effective exponent $\alpha \approx 0.89$. Such a value which is smaller than unity can be understood as coming from the logarithmic factor.

Another test on the growing length scale in the unfrustrated array can be performed by calculating the equaltime phase correlation function

$$
C_{s}(r, t) \equiv \frac{1}{L^{2}} \sum_{i}\left\langle\cos \left[\phi_{i+\mathbf{r}}(t)-\phi_{i}(t)\right]\right\rangle .
$$

Figure 2 shows the equal-time spatial correlation function of phases at temperature $T=0.45$. Behavior of the phase correlation function $C_{s}(r, t)$ with distance $r$ is displayed in Fig 2 at various time stages $t=8$ to 2048 . Critical dynamic scaling has been attempted with various values of $\eta$ : For each trial value of $\eta$, the rescaled function $\tilde{C}_{s}(r, t) \equiv r^{\eta} C_{s}(r, t)$ has been plotted in terms of the rescaled distance $r / \ell_{s}$, where the length scale $\ell_{s}$ as a function of time $t$ has been obtained from the condition $\tilde{C}_{s}\left(r=\ell_{s}, t\right)=C_{0} \equiv 0.4$. The value of $\eta$ yielding the best scaling collapse has been chosen, which is shown in Fig. 2(b). The resulting optimal value $\eta=0.083$ is in fair agreement with the value from the theoretically predicted form of the equilibrium correlation exponent 18 $\eta_{e q} \approx T / 2 \pi+T^{2} / 4 \pi \approx 0.0877$. The length scale $\ell_{s}$ obtained in this way is presented in Fig. 2(c), where one 


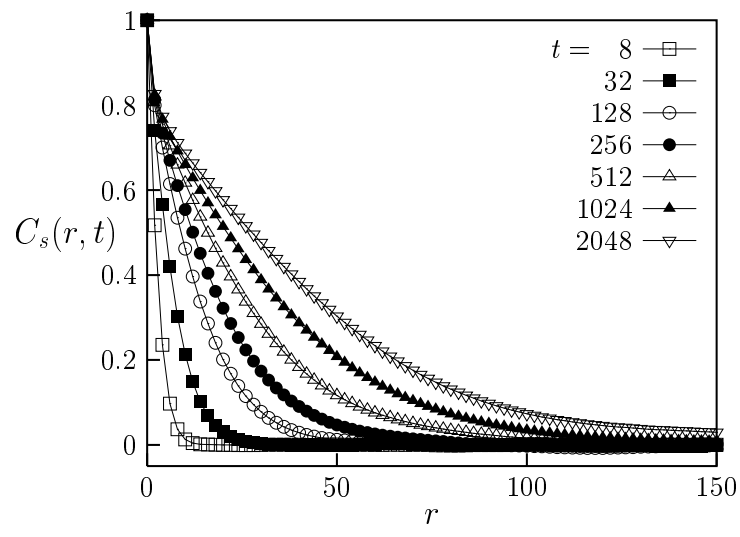

(a)

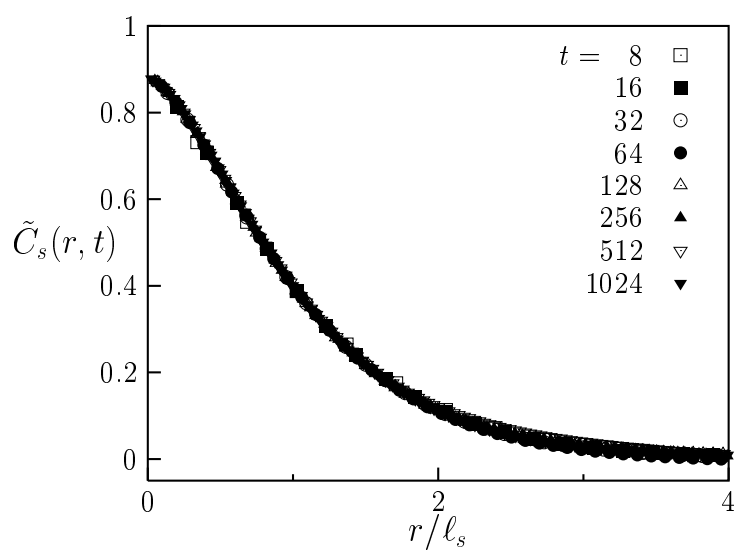

(b)

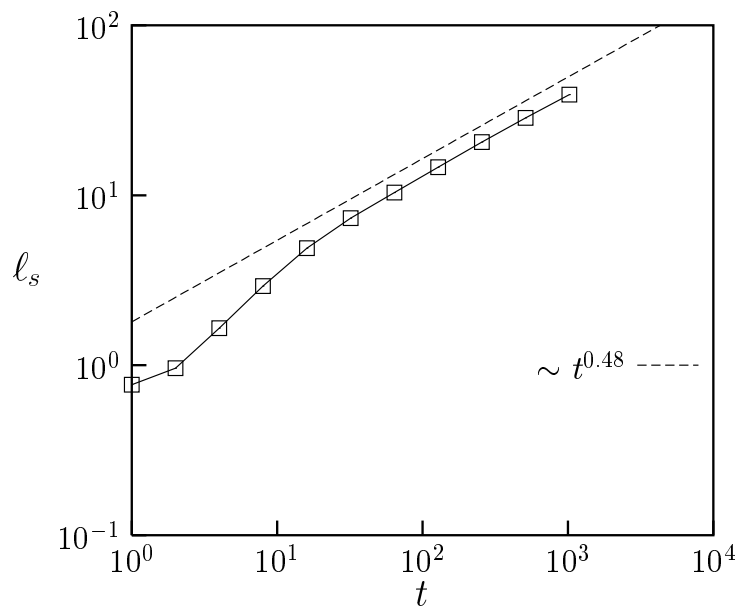

(c)

FIG. 2: (a) Spatial behavior of the equal-time phase correlation function at various time stages in the unfrustrated array of size $L=400$ at temperature $T=0.45$. (b) Scaling collapse of the data in (a), plotted in terms of the rescaled correlation function with $\eta=0.083$ and the scaling length $\ell_{s}$ defined in the text. (c) Scaling length versus time at $T=0.45$, exhibiting power-law growth $\ell_{s} \sim t^{0.48}$. finds that $\ell_{s} \sim t^{1 / z_{s}}$ with $1 / z_{s} \approx 0.48$, slightly below the expected value $1 / 2$. We have also performed simulations at various temperatures, and found that the value of $1 / z_{s}$ is nearly constant, showing only small variations in the range $1 / z_{s} \approx 0.47$ to 0.48 . The reason behind this small but apparently systematic deviation is not clear at this stage. Presumably, it is related to the fact that we are working with a lattice system where small local barriers exist for the motion of point vortices. At the early-time stage when the vortices are close together, these barriers are negligible compared with inter-vortex forces; at the late-time stage when vortices are far away, local energy barriers are likely to hinder appreciably the vortex motion, possibly resulting in smaller values of the effective growth exponent.

\section{COARSENING IN THE RELAXATION DYNAMICS OF FULLY FRUSTRATED ARRAYS}

We now consider the fully frustrated array $(f=1 / 2)$ with the gauge field:

$$
A_{i j}=\left\{\begin{array}{cl}
0 & \text { for } \mathbf{r}_{j}=\mathbf{r}_{i}+\hat{\mathbf{x}} \\
\pi x_{i} & \text { for } \mathbf{r}_{j}=\mathbf{r}_{i}+\hat{\mathbf{y}}
\end{array}\right.
$$

which corresponds to the system of $X Y$ spins with one antiferromagnetic coupling per plaquette. The fully frustrated array has the discrete $\mathrm{Z}_{2}$ symmetry in addition to the underlying $\mathrm{U}(1)$ symmetry, yielding the antiferromagnetic long-range order in the chirality at temperatures $T<T_{c}(\approx 0.45) .9$ Due to the existence of the chirality order parameter, the coarsening dynamics in a fully frustrated array proceeds in a manner quite different from that of the unfrustrated array.

In order to probe nonequilibrium coarsening of a fully frustrated array, we measure the equal-time spatial correlations of the phase and the chirality. The phase correlation function in the fully frustrated array can be defined in the same way as Eq. (20) for the unfrustrated array, only if it is assumed that the correlations are measured only for even lattice spacings along both the axes. Restricting the position vectors to those with components of even lattice spacings corresponds to forming a superlattice, each plaquette of which consists of four plaquettes of the original lattice. Such a superlattice system yields a ferromagnetic ground state within itself at zero temperature. Meanwhile, the chirality correlation function is defined as follows:

$$
C_{q}(r, t) \equiv \frac{1}{N^{2}} \sum_{\mathbf{R}}(-1)^{x+y}\left\langle q_{\mathbf{R}+\mathbf{r}}(t) q_{\mathbf{R}}(t)\right\rangle,
$$

where the chirality

$$
q_{\mathbf{R}}(t) \equiv \operatorname{sgn} \sum_{P} \sin \left[\widetilde{\phi}_{i j}(t)-\mathbf{r}_{i j} \cdot \boldsymbol{\Delta}(t)\right],
$$

with $\sum_{P}$ denoting the directional plaquette summation of links around dual lattice site $\mathbf{R}$, describes the degrees 


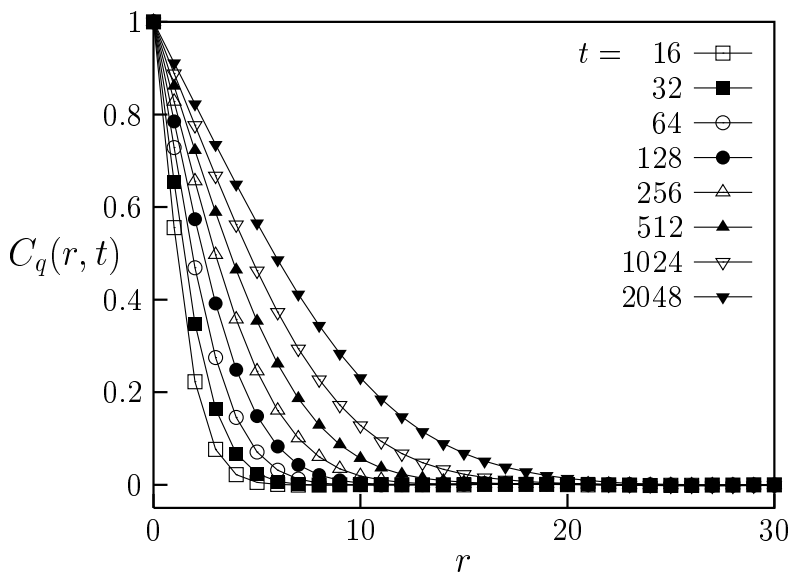

(a)

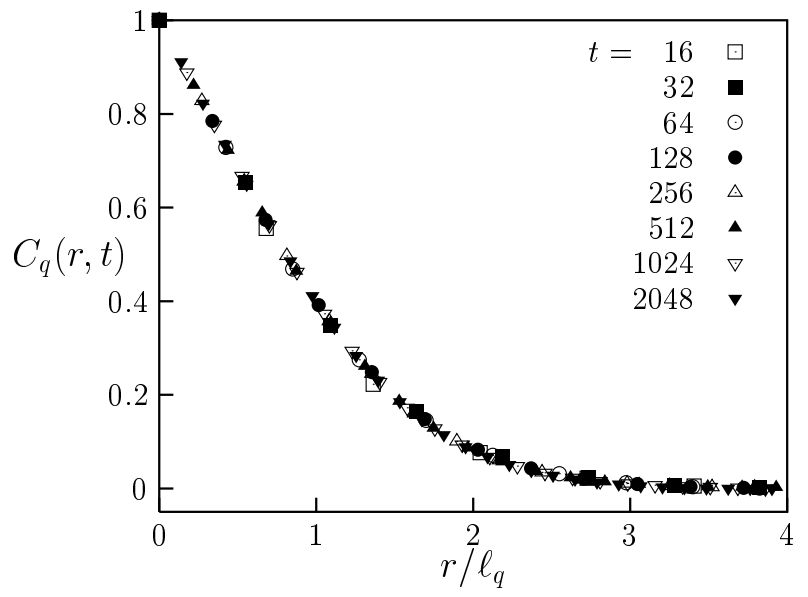

(b)

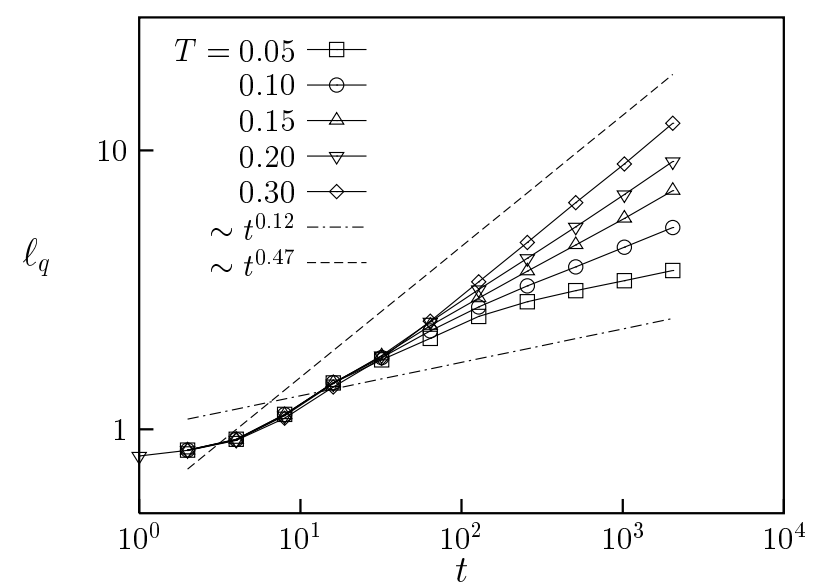

(c)

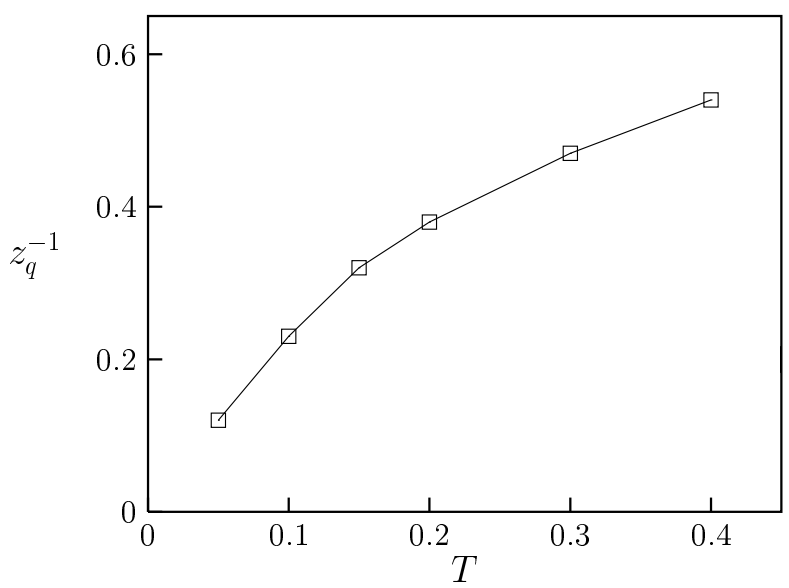

(d)

FIG. 3: (a) Spatial behavior of the equal-time chirality correlation function at various time stages in the fully frustrated array of size $L=128$ at temperature $T=0.15$. (b) Scaling collapse of the data in (a) with the appropriate scaling length $\ell_{q}$. (c) Scaling length versus time at various temperatures $T=0.3,0.2,0.15,0.1,0.05$ from above, revealing temperature-dependent growth. (d) Growth exponent versus temperature, manifesting that growth becomes slower as the temperature is lowered.

of freedom associated with the $\mathrm{Z}_{2}$ symmetry. The factor $(-1)^{x+y}$, in the definition of $C_{q}(r, t)$, where $\mathbf{r} \equiv(x, y)$, takes care of the staggered nature of the chirality ordering. Due to the existence of these two kinds of degrees of freedom, the relaxation and ordering process proceeds via annihilation of two kinds of defects i.e., point defects (tending to restore the $\mathrm{U}(1)$ symmetry) and line defects (domain walls tending to restore the chiral $Z_{2}$ symmetry). Point defects correspond to the corners of line defects where fractional charges of magnitude $1 / 4$ reside. 19 Hence the annihilation processes of these two kinds of defects are closely coupled with each other. Note that conventional point vortices with integer charges, possessing high energies, are annihilated very quickly in the early stage of coarsening; thus they are irrelevant in the latetime coarsening behavior of the system.

Here we present our simulation results for the ordering dynamics of only the chirality since the phase ordering exhibits similar features except for the additional $\eta$ expo- nent due to the criticality. Figure 3 shows the equal-time correlation function $C_{q}(r, t)$ of the (staggered) chirality at temperature $T=0.15$. Spatial behavior of $C_{q}(r, t)$ is exhibited in Fig. 3(a) at various time stages $t=16$ to 2048. Scaling of the correlations can be obtained similarly to the case of the unfrustrated array, except for the obvious fact that $\eta_{q}=0$, reflecting the emergence of true long-range order. The resulting nice scaling collapse is shown in Fig. 3(b). The scaling length $\ell_{q}$ is plotted versus time in Fig. 3(c) at various temperatures, from which one obtains the relation $\ell_{q} \sim t^{1 / z_{q}}$ with the appropriate growth exponent $1 / z_{q}$.

In contrast to the case of the unfrustrated array, the growth exponent in the fully frustrated array depends strongly on the temperature, as shown in Fig. 3 (d). For example, we have the value $1 / z_{q} \approx 0.38$ at temperature $T=0.2$ but it decreases to $1 / z_{q} \approx 0.32$ at $T=0.15$. At low temperatures, in particular, $1 / z_{q}$ depends almost linearly on the temperature, which has also been ob- 


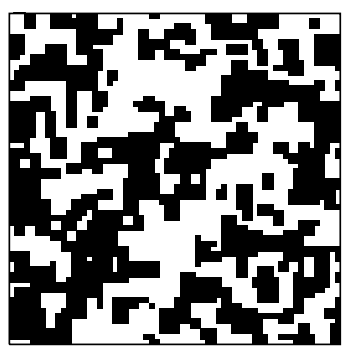

(a)

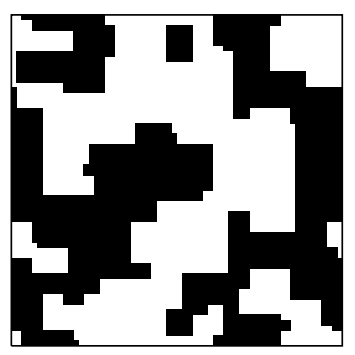

(b)

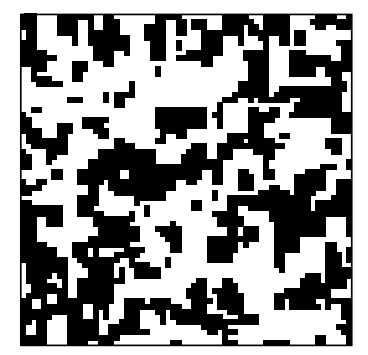

(c)

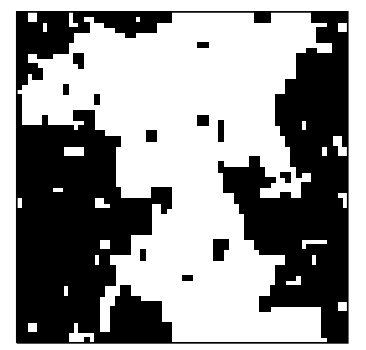

(d)
FIG. 4: Snapshots of the configuration of the staggered chirality in the fully frustrated array of size $L=64$ at temperature $T$ and time $t$. (a) $T=0.1$ and $t=32$; (b) $T=0.1$ and $t=1024$; (c) $T=0.4$ and $t=32$; (d) $T=0.4$ and $t=1024$.

served in the ordering dynamics of the fully frustrated $X Y$ model based on the simple Langevin equation.11 On the other hand, the temperature dependence of the domain growth emerges only after some time $\left(t \gtrsim t_{q} \approx 30\right)$; before that time $\left(t \lesssim t_{q}\right)$ the domain growth apparently does not change with the temperature. Such temperature independence at the early-time stage is a distinctive feature that is not found in the simple Langevin dynamics. This indicates that dissipation at the early-time stage is dominated by a mechanism that is different from the temperature-dependent activation dynamics operating at the late-time stage.

In order to help understand the temperaturedependence of the dynamic exponent, we have also examined how ordering proceeds with time. Shown in Fig. 1 are the snapshots at various time instants of ordering configurations in terms of the staggered chirality. Figure 1 (a) and (b) exhibit the ordering process for quenching to low temperatures $(T=0.1)$ while $(\mathrm{c})$ and $(\mathrm{d})$ to higher temperatures $(T=0.4)$, where black and white regions represent domains with opposite (staggered) chirality ordering. One can observe distinctive features in the domain growth morphology in the two cases: At low temperatures, typical separation between consecutive corner defects along a domain wall grows larger in time. At higher temperatures, in contrast, the typical separation between corners remains more or less constant throughout the ordering process. Slow ordering at low temperatures $(T=0.1)$ compared with that at high temperatures $(T=0.4)$ can also be observed here, in agreement with the apparent temperature-dependence of $1 / z_{q}$ obtained from the scaling analysis of the chirality correlation function.

The above morphological characteristics are related to the time dependence of the number $N_{p}$ of point defects and of the total length $N_{l}$ of line defects. Simulations reveal power-law relaxation toward equilibrium with temperature-dependent exponents, as shown Fig. 5(a) to (c). Namely, we have $\tilde{N}_{p}(t) \equiv N_{p}(t)-N_{p}(\infty) \sim t^{-\nu_{p}}$ and $\tilde{N}_{l}(t) \equiv N_{l}(t)-N_{l}(\infty) \sim t^{-\nu_{l}}$, where $N_{p}(\infty)$ and $N_{l}(\infty)$ are the values in equilibrium. These equilibrium values, which depend on the temperature, can easily be obtained from dynamic simulations, beginning with one of the the known ground state configurations. The resulting equilibrium values are negligibly small at low temperatures $(T \lesssim 0.2)$ and the total amounts $N_{p}$ and $N_{l}$ as well as the excess amounts $\tilde{N}_{p}$ and $\tilde{N}_{l}$ display power-law decay. The corresponding decay exponents together with $1 / z_{q}$ are shown in Fig. 5(d) as functions of the temperature. At low temperatures, we have $\nu_{p}>\nu_{l}$ and point defects decay away faster than line defects, resulting in growing separation between corner defects along domain walls. At higher temperatures, on the other hand, we have $\nu_{p} \approx \nu_{l}$, and the average distance between neighboring point defects along a line defect does not change appreciably with time, similarly to the previous works 1122 , which argued, based on such numerical results, that there exists a roughening transition at a finite temperature. However, it was argued in a recent work that due to the finite excitation energy for the double-step kinks, the domain walls should be rough at any finite temperature.20 If this is indeed the case, asymptotic decay exponents for the domain walls and corner charges should be the same at all finite temperatures. Unfortunately, numerical confirmation of this would be very formidable due to the slow domain growth at low temperatures.

\section{SUMMARY}

We have studied the non-equilibrium relaxation and coarsening dynamics of two-dimensional superconducting arrays in the overdamped limit under zero external driving current. In the case of unfrustrated arrays, we find that the friction constant of a vortex remains finite in the limit of large extent of the vortex; this is argued to lead to the absence of logarithmic factors in the length scale and in the vortex number decay, which is in reasonable agreement with simulation results. In the case of fully frustrated arrays, relaxation and coarsening dynamics are characterized by decay of line defects (chirality domain walls) as well as of point defects (corner charges). Here strong temperature dependence is found in the characteristics of the late-time domain growth, which can be explained by activated domain wall dynamics across the energy barriers with logarithmic size dependence. In the early-time regime, on the other handr, there exists a relatively long period of transient dynamics which is independent of the temperature; this may be attributed to 


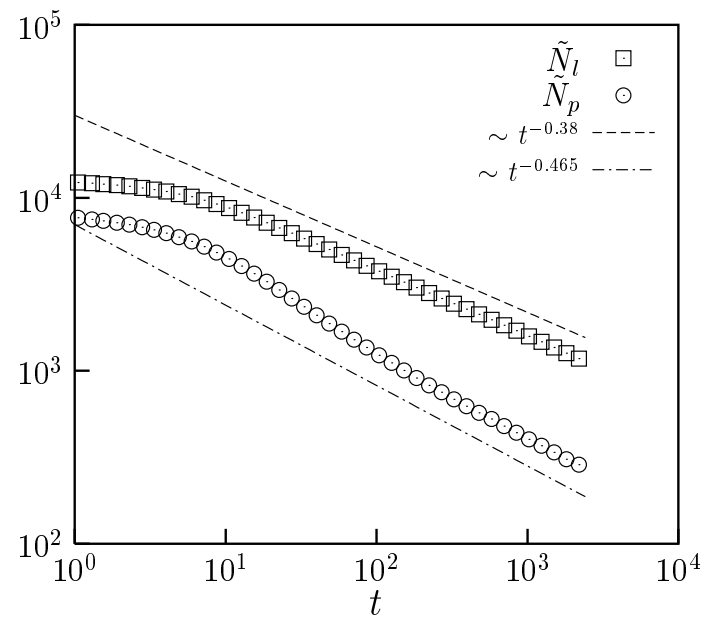

(a)

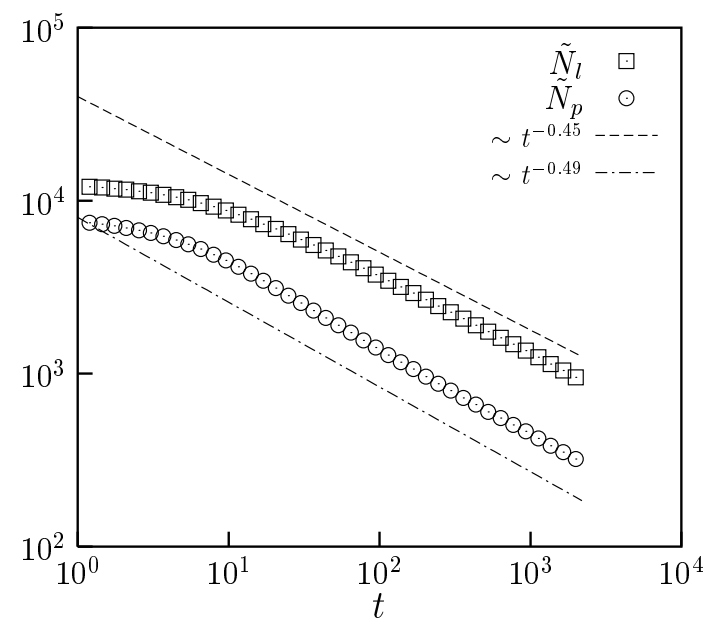

(b)

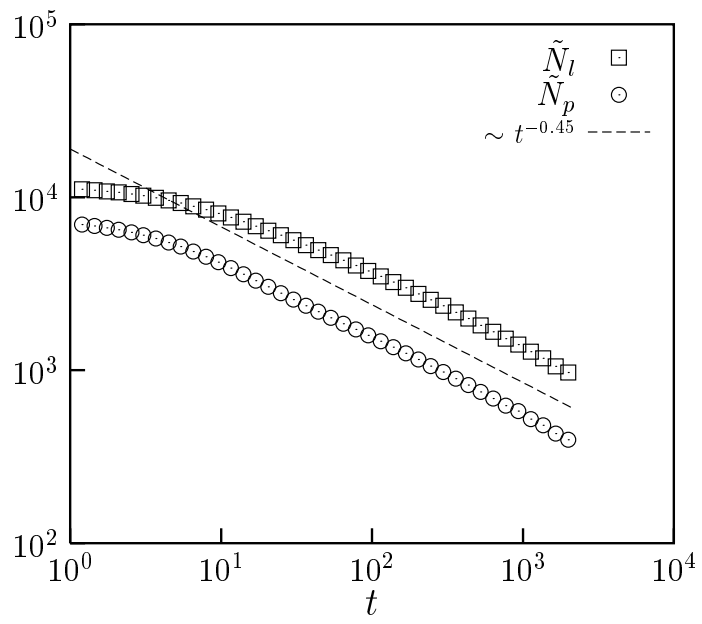

(c)

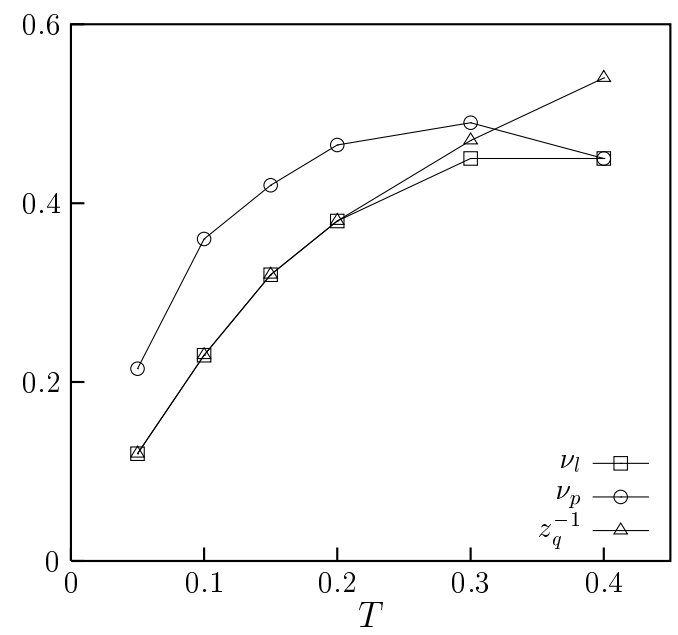

(d)

FIG. 5: Relaxation of the excess length of the line defects and of the excess number of point defects associated with staggered chirality domains in the array of size $L=128$ at temperature (a) $T=0.20$; (b) $T=0.30$; (c) $T=0.40$. Shown in (d) is the temperature-dependence of the decay exponents $\nu_{l}$ and $\nu_{p}$ for line defects and for point defects, respectively, together with the chirality growth exponent $1 / z_{q}$.

the effects of initial random distributions of the strong Lorentz force, making the thermal noise irrelevant. It would be of interest to investigate the coarsening dynamics of Josephson-junction arrays under external magnetic fields and to compare with our simulation results.

\section{Acknowledgments}

We thank Bongsoo Kim for helpful discussions. This work was supported in part by the Korea Research Foun- dation through Grant No. 2000-015-DP0138.
* Also at Korea Institute for Advanced Study, Seoul 130-012, Korea.

1 A. J. Bray, Adv. Phys. 43, 357 (1994).
2 R. Loft and T. A. DeGrand, Phys. Rev. B 35, 8528 (1987); H. Toyoki and K. Honda, Prog. Theor. Phys. 78, 237 (1987). 
3 M. Mondello and N. Goldenfeld, Phys. Rev. A 42, 5865 (1990); Phys. Rev. E 47, 2384 (1993).

4 A. J. Bray and K. Humayun, J. Phys. A 23, 5897 (1990); S. Puri and C. Roland, Phys. Lett. A 151, 500 (1990); H. Toyoki, Phys. Rev. A 42, 911 (1990).

5 B. Yurke, A. N. Pargellis, T. Kovacs, and D. A. Huse, Phys. Rev. E 47, 1525 (1993); R. E. Blundell and A. J. Bray, ibid. 49, 4925 (1994); J.-R. Lee, S. J. Lee, and B. Kim, ibid. 52, 1550 (1995).

6 S. Teitel and C. Jayaprakash, Phys. Rev. B 27, 598 (1983); M.Y. Choi and S. Doniach, ibid. 31, 4516 (1985).

7 See, e.g., C. Giovannela and M. Tinkham, (eds.) Macroscopic Quantum Phenomena and Coherence in Superconducting Networks (World Scientific, Singapore, 1996); Physica B 222, 253 (1996).

8 V.L. Berezinskii, Zh. Eksp. Teor. Fiz. 59, 907 (1970) [Sov. Phys. JETP 32, 493 (1971)]; J.M. Kosterlitz and D.J. Thouless, J. Phys. C 6, 1181 (1973); J.M. Kosterlitz, ibid. 7, 1046 (1974).

9 J. Lee, J.M. Kosterlitz, and E. Granato, Phys. Rev. B 43, 11531 (1991); G. Ramirez-Santiago and J.V. José, Phys. Rev. Lett. 68, 1224 (1992); S. Lee and K.-C. Lee, Phys. Rev. B 49, 15184 (1994); P. Olsson, Phys. Rev. Lett. 75,
2758 (1995); G.S. Jeon, S.Y. Park, and M.Y. Choi, Phys. Rev. B 55, 14088 (1997).

10 S. E. Korshunov, Phys. Rev. B 50, 13616 (1994).

11 S. J. Lee, J.-R. Lee, and B. Kim, Phys. Rev. E 51, R4 (1995).

12 J.-R. Lee, S. J. Lee, B. Kim, and I. Chang, Phys. Rev. Lett. 79, 2172 (1997).

13 B.J. Kim, P. Minnhagen, and P. Olsson, Phys. Rev. B 59, 11506 (1999).

14 H. Weber, P. Minnhagen, Phys. Rev. B 37, 5986 (1988).

15 G. Ryskin and M. Kremenetsky, Phys. Rev. Lett. 67, 1574 (1991); A. J. Bray, A. J. Briant, and D. K. Jervis, ibid. 84, 1503 (2000); A. J. Bray, Phys. Rev. E 62, 103 (2000).

16 F. Rojas and A. D. Rutenberg, Phys. Rev. E 60, 212 (1999).

17 S. R. Shenoy, J. Phys. C 18, 5163 (1985).

18 J. Villain, J. Phys. (Paris) 36, 581 (1973).

19 T. C. Halsey, J. Phys. C 18, 2437 (1985); S. E. Korshunov and G. V. Uimin, J. Stat. Phys. 43, 1 (1986); S. E. Korshunov, ibid. 43, 17 (1986).

20 S. E. Korshunov, Phys. Rev. Lett. 88, 167007 (2002). 